\title{
Recent advances in hydrogels in terms of fast stimuli responsiveness and superior mechanical performance
}

\author{
Abu Bin Imran, Takahiro Seki and Yukikazu Takeoka \\ This review addresses the potential development of polymer hydrogels in terms of fast stimuli responsiveness and superior \\ mechanical properties. The slow response and mechanical weakness of stimuli-sensitive hydrogels have been considered as the \\ main barriers for their further development and practical application. It has been a dream of scientists to have fast stimuli- \\ responsive hydrogels with superior mechanical performance. In this review, the principal reasons behind the poor stimulus \\ sensitivity and mechanical strength of polymer gels are highlighted, and we present some pioneering physical and chemical \\ efforts aimed at fabricating hydrogels with high stimuli sensitivities and/or superior mechanical properties.
}

Polymer Journal (2010) 42, 839-851; doi:10.1038/pj.2010.87; published online 29 September 2010

Keywords: butterfly pattern; fast stimuli sensitivity; hydrogel; LCST; mechanical property

\section{INTRODUCTION}

Polymer hydrogels (hereafter called 'hydrogels') are three-dimensional polymer networks in which the voids are filled with water. These hydrogels are widely used in a variety of industrial and consumer products such as oil dewatering systems, mechanical absorbers, diapers and contact lenses. One of the most remarkable areas of research on hydrogels in the past few decades has been their stimulus sensitivity. Hydrogels composed of stimuli-responsive polymers reversibly alter their shape and volume in response to small variations in the environment, such as $\mathrm{pH}$, temperature, light and electric and magnetic fields, thereby changing their physico-chemical characteristics. ${ }^{1}$ Among all of the available environmentally responsive hydrogels, temperature-responsive hydrogels have attracted the most attention because of the facile tuning of their properties. In particular, poly $(\mathrm{N}$ isopropylacrylamide) (poly(NIPA)) hydrogels have been widely investigated as thermosensitive hydrogels. Poly(NIPA) has a low critical solution temperature (LCST) at around $32{ }^{\circ} \mathrm{C}$ in water. The flexible coil of poly(NIPA) (soluble) converts into a compact globular state (insoluble) at the LCST, and this conformational change is reversible. ${ }^{2}$ Thus, hydrogels composed of poly(NIPA) undergo a reversible volume change at a similar transition temperature in water. Poly(NIPA) hydrogels have potential applications in drug delivery systems, therapeutic agents, diagnostic devices, biomaterials, cell cultivation, biocatalysts, sensors, microfluidic devices, actuators, optical devices and size-selective separation among others. ${ }^{3-5}$

A recent subject that has attracted much attention in the field of gel science is the fabrication of hydrogels with the rapid stimuli responsiveness and superior mechanical properties required for many applications of stimuli-responsive hydrogels. The creation of durable hydrogels that exhibit reversible and rapid changes in shape or volume, the likes of which are found in living organisms such as muscle, has been a challenge for materials chemists. Hydrogels synthesized from monomer solutions by radical polymerization, a general-purpose method for making synthetic hydrogels, however, face several constraints. The inherently weak mechanical properties caused by underlying spatial inhomogeneity during polymerization and extremely slow responsiveness caused by critical slowing down and vitrification during the shrinking process restrict the widespread use of these hydrogels. ${ }^{6}$ Although some approaches have been developed to improve the stimuli sensitivities and mechanical properties of hydrogels, it remains a challenge to design ideal gel networks with a combination of desired properties.

In this review, we will begin by introducing some pioneering work in the synthesis of hydrogels with fast stimuli responsiveness specifically attributed to chemical modifications. Work based on thermosensitive NIPA as a monomer has been the main focus of attention. Although hydrogels synthesized through these techniques improve stimuli sensitivity to a greater extent, in most cases, not enough attention has been given to the mechanical properties of these hydrogels, and these properties have not been improved. We will therefore review some recently developed novel methods to improve the mechanical properties of hydrogels and discuss the application of these methods for preparing stimuli-responsive hydrogels with superior mechanical performance.

\section{FAST STIMULI-SENSITIVE HYDROGELS}

The volume phase transition ${ }^{7-9}$ of hydrogels between the swollen state and collapsed state in response to various types of stimuli indicates 
that hydrogels can be used as smart materials. Here, the term 'smart' refers to materials that are sensitive to changes in the environment. Unfortunately, however, the slow response of hydrogels, which is an inherent characteristic of the volume phase transition phenomenon caused by a critical slowing down, has prevented them from being developed for new technologies. Moreover, vitrification during the shrinking process also contributes to the slow change in volume. After a temperature jump, a dense skin layer forms on the surface of poly(NIPA) hydrogels that inhibits water loss from the inner portion and, consequently, slows down the volume change. ${ }^{10}$ Without the volume phase transition, in which the volume change of hydrogels is drastic but continuous, the characteristic duration of the volume change can be much shorter than in a discontinuous system. The relaxation time of the volume change of hydrogels, however, is highly dependent on the size of the hydrogel, as the swelling and deswelling of hydrogels is a diffusion process. According to the Tanaka-Fillmore theory, the shrinking rate is inversely proportional to the square of the smallest spatial dimension of the hydrogel. As a result, large, bulky hydrogels typically exhibit slow changes in volume. ${ }^{6}$

To date, many physical processes have been developed to obtain high thermosensitivity in poly(NIPA) hydrogels, including the following: preparations of phase-separated heterogeneous structures ${ }^{11,12}$ and macroporous or mesoporous structures, ${ }^{13-15}$ gel formation via vacuum synthesis, ${ }^{16}$ as well as freezing techniques. ${ }^{17,18}$ As an example, a poly(NIPA) hydrogel with a phase-separated heterogeneous network structure can be prepared by polymerization at a temperature above the LCST or in particular mixed solvents, including solvents composed of $\mathrm{H}_{2} \mathrm{O}$ and an organic solvent such as acetone, phenol or tetrahydrofuran. A pore-forming component such as sodium chloride, glucose, poly(ethylene glycol) (PEG), $\mathrm{SiO}_{2}$ or a hydrophilic or hydrophobic additive can be introduced into the pregel solution. The subsequent removal of this pore-forming component from the hydrogel network provides a macroporous network with fast thermoresponsiveness. The basic mechanism of all these processes involves the effect of micro- or macro-level manipulated structures, which are much larger than molecular level structures, on the quick volume change by the expulsion or absorption of water molecules from or into the hydrogel network. These processes, however, are not suited for some practical applications, as the hydrogels lose their mechanical strength, toughness and optical transparency because of their spongy structures. We will not further discuss such porous hydrogels; rather, we will primarily focus on the strategies used to obtain fast thermoresponsive hydrogels using chemical techniques by which the gel networks are modified at the molecular level. Some of the basic techniques used are mentioned below.

\section{Comb-type poly(NIPA)-grafted gels}

Yoshida et al. prepared comb-type poly(NIPA)-grafted gels as follows. First, a chain transfer agent was used to synthesize various linear poly(NIPA)s with different molecular weights and one amino end group. Next, the amino group was converted into an acrylate group that could polymerize with other vinyl monomers. Finally, the semitelechelic linear poly(NIPA)s with acrylate end groups were polymerized with NIPA and small amounts of a crosslinker. Poly (NIPA) chains with one freely mobile side end were thereby grafted onto the polymer networks (Figure 1a). ${ }^{19,20}$ These grafted polymer chains can respond faster than crosslinked poly(NIPA) chains and form hydrophobic nuclei in response to a rise in temperature (Figure 1b). The aggregated nuclei then form many channels for the diffusion of water to enhance the shrinking rate of the crosslinked a
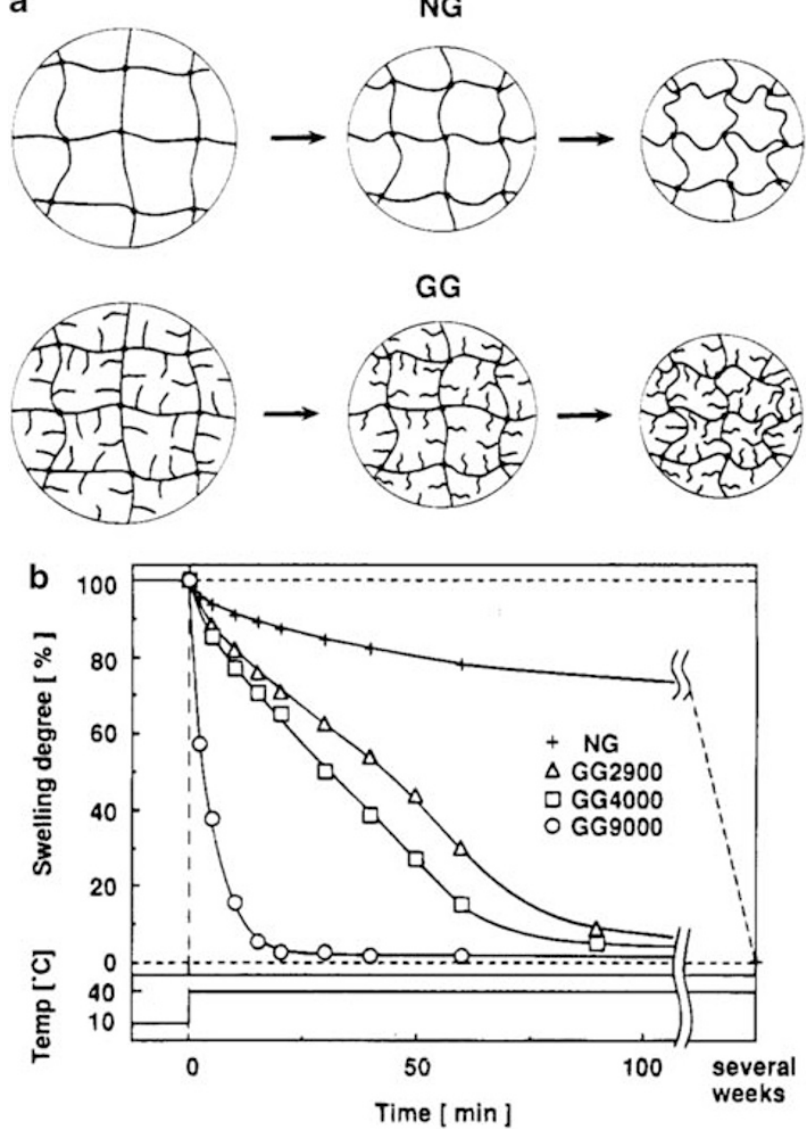

Figure 1 (a) Schematic illustration of the deswelling kinetics of hydrogels shrinking beneath their phase-transition temperatures. (b) Time course of deswelling for hydrogels undergoing shrinking at $40^{\circ} \mathrm{C}$ in response to a stepwise temperature change from $10^{\circ} \mathrm{C}$. NG: traditional poly ( $N$-isopropylacrylamide) (PNIPA) hydrogel; GG: comb-type grafted PNIPA hydrogel; 2900, 4000 and 9000 are the molecular weights of the grafted chains. (Reproduced with permission from Kaneko et al. ${ }^{20}$ )

segment of the hydrogel with respect to non-grafted traditional poly(NIPA) hydrogels.

Similarly, the application of a living radical polymerization method to prepare a comb-type grafted polymer network significantly enhances the deswelling response of poly(NIPA) gels. ${ }^{21,22}$ Liu et al. ${ }^{21}$ used reversible addition-fragmentation chain transfer polymerization to synthesize a functional poly(NIPA) hydrogel (Figure 2). This hydrogel consisted of dangling chains, mainly caused by a chain transfer reagent that markedly retarded the crosslinking reaction and showed accelerated shrinking kinetics and higher swelling ratios compared with conventional hydrogels.

Poly(NIPA) hydrogels with micellar structures for water pathways Noguchi et al. ${ }^{23}$ grafted surfactants capable of micelle formation on the polymer network of a poly(NIPA) gel (Figure 3a). These grafted surfactants form micelle structures at temperatures above the LCST (Figure $3 \mathrm{~b}$ ). In the shrinking process, trapped water molecules are quickly squeezed out through the hydrophilic channels between the hydrophilic outer shells of the micelles; consequently, the hydrogel shrinks rapidly. A pendant micelle structure can also be introduced into the polymer chains to improve their thermosensitivity. ${ }^{24}$ 
<smiles>C=CC(=O)NC(C)C</smiles><smiles>CC(C#N)(CCC(=O)O)SC(=S)c1ccccc1</smiles>

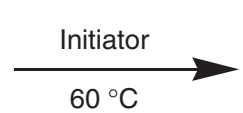<smiles>C=CC(=O)NC(C)C</smiles>

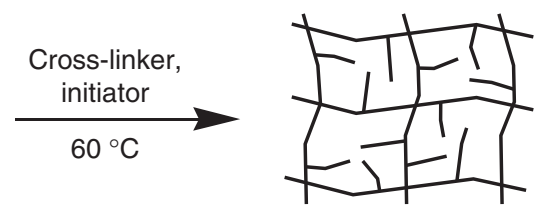

Figure 2 Synthetic procedure for comb-type grafted hydrogels by reversible addition-fragmentation chain transfer. (Reproduced with permission from Liu et al. ${ }^{21}$ )

a

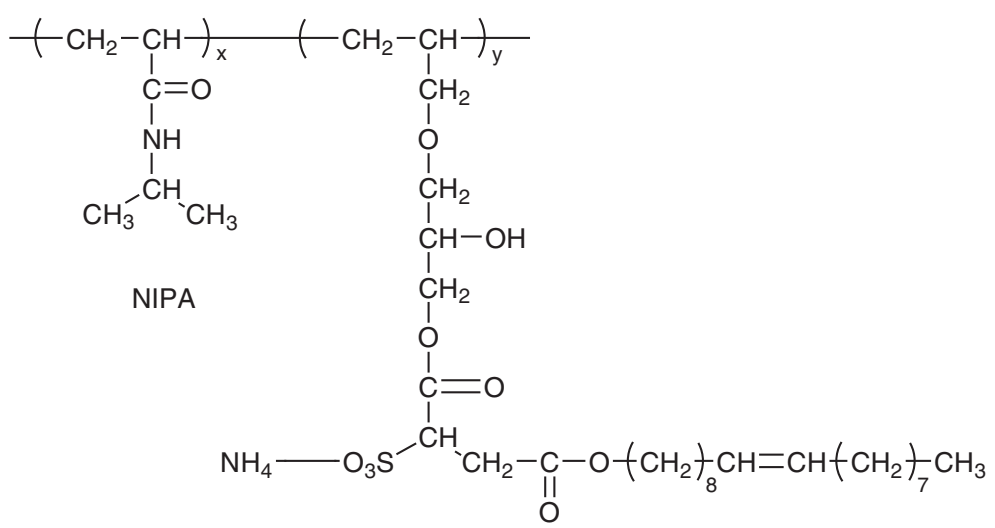

Grafted surfactant

b

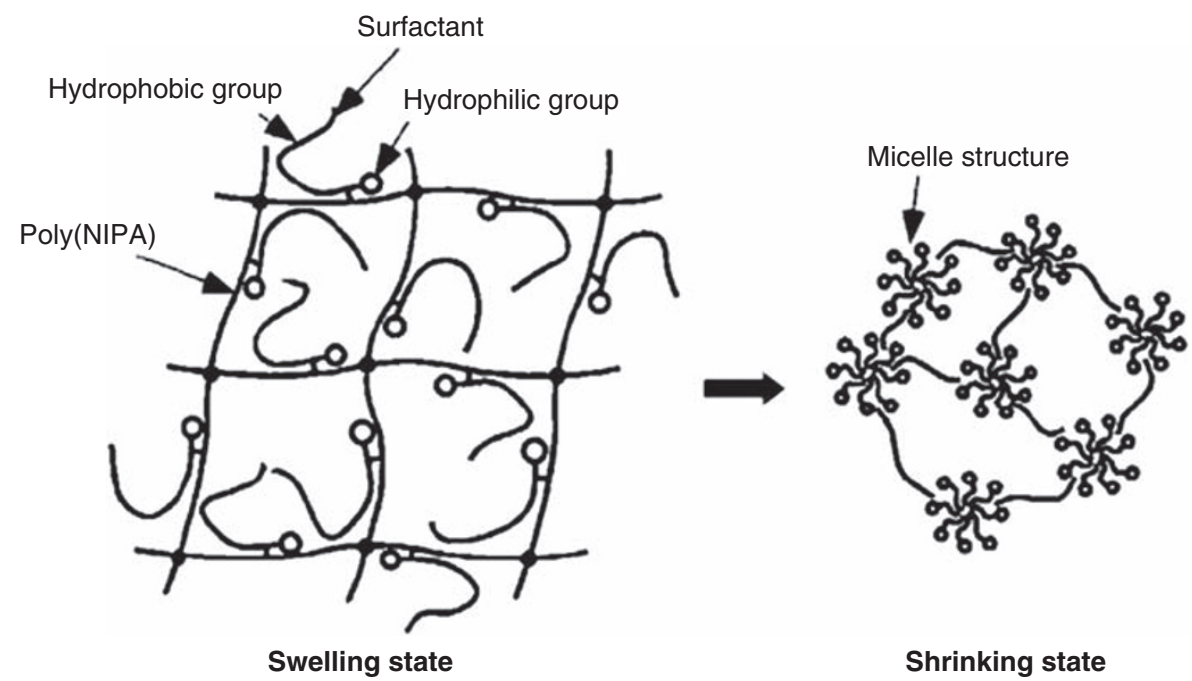

Figure 3 (a) Chemical structure of poly(N-isopropylacrylamide) (poly(NIPA)-co-surfactant monomer. (b) Shrinking mechanism of a poly(NIPA-co-surfactant monomer) gel in response to increasing temperature. (Reproduced with permission from Noguchi et al. ${ }^{23}$ )

Interpenetrating polymer network gels with low skin layer formation Gil and Hudson ${ }^{25}$ developed an interpenetrating polymer network (IPN) of silk fibronin/poly(NIPA). The silk fibronin reduces skin layer formation; consequently, a fast deswelling rate is observed for the hydrogel (Figure 4). Poly(NIPA) hydrogels with an IPN structure exhibit slightly improved mechanical strength: The compression 
modulus and tensile modulus of IPN-poly(NIPA) hydrogels are, respectively, 3.5 and 16.8 times higher than those of conventional poly(NIPA) hydrogels. ${ }^{26}$ A semi-IPN created by combining poly (acrylic acid) and a porous poly(NIPA) hydrogel also shows fast responses to both temperature and $\mathrm{pH}$ changes. ${ }^{27}$ If a small amount of hydrophilic moieties is introduced into the hydrogel network,
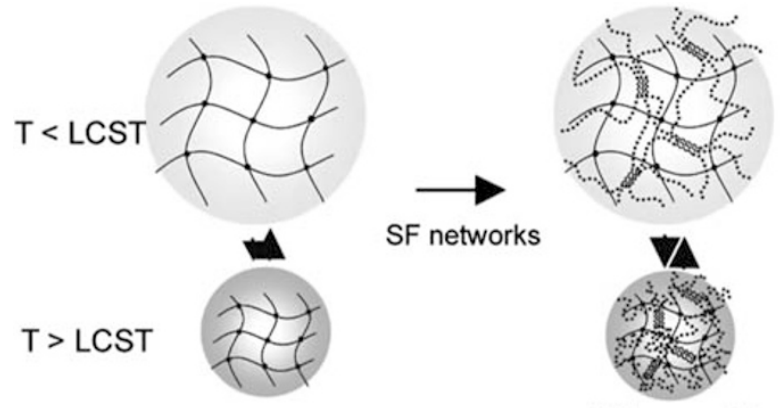

Higher modulus

Faster deswelling
Figure 4 Illustration of the interpenetrating polymer network (IPN) of silk fibronin/poly(N-isopropylacrylamide) (poly(NIPA)). The poly(NIPA) network undergoes expansion/aggregation in response to temperature, whereas the $\beta$-sheet structure of fibronin remains intact and enhances the mechanical properties of the hydrogel network. (Reproduced with permission from Gil and Hudson. ${ }^{25}$ ) hydrophobic aggregation on the hydrogel surface decreases; consequently, the formation of a thick, impermeable skin layer is protected to give fast thermoresponsiveness. ${ }^{28,29}$ When large amounts of hydrophilic moieties are used, however, the hydrogel drastically loses its deswelling kinetics.

As mentioned above, the formation of stimuli-sensitive water pathways and the inhibition of skin layer formation during the contraction of hydrogels are the primary ways used to obtain fast stimuli-responsive hydrogels. All of the fast stimuli-responsive hydrogels discussed above have the potential to be smart hydrogels, but their mechanical properties, homogeneity, biocompatibility and reversibility need to be improved for applications that require mechanical durability.

\section{HYDROGELS WITH STRONG MECHANICAL PROPERTIES}

Free-radical polymerization of a random crosslink between monomers and crosslinkers results in both lightly and heavily crosslinked regions in gel networks, and these different types of regions introduce spatial inhomogeneity into hydrogels. Chemical crosslinks fix the polymer chains to different segments with different lengths. Under deformation, most of the stress is thereby localized on the shortest polymer chains, and soon the crosslinked polymer networks split into several pieces. This mechanical weakness at the molecular level is also sustained at the macro level; thus, conventional hydrogels are mechanically fragile by nature. Recently, various research groups have reported mechanically superior hydrogels. In this section, these mechanically superior hydrogels will be reviewed briefly.

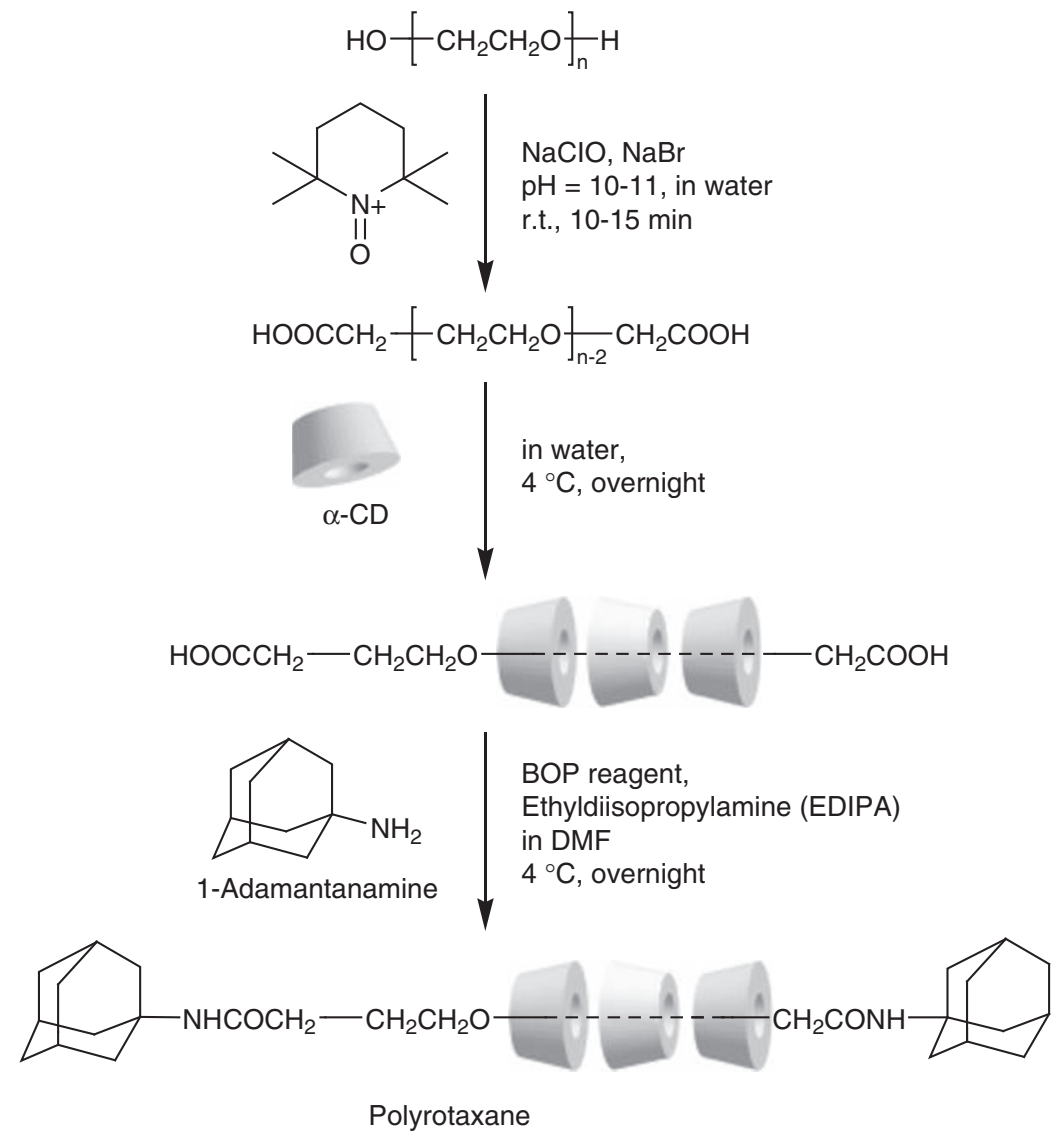

Figure 5 Preparation of polyrotaxane from poly(ethylene glycol), $\alpha$-cyclodextrin and 1-adamantanamine. (Reproduced with permission from Araki et al. ${ }^{33}$ ) 

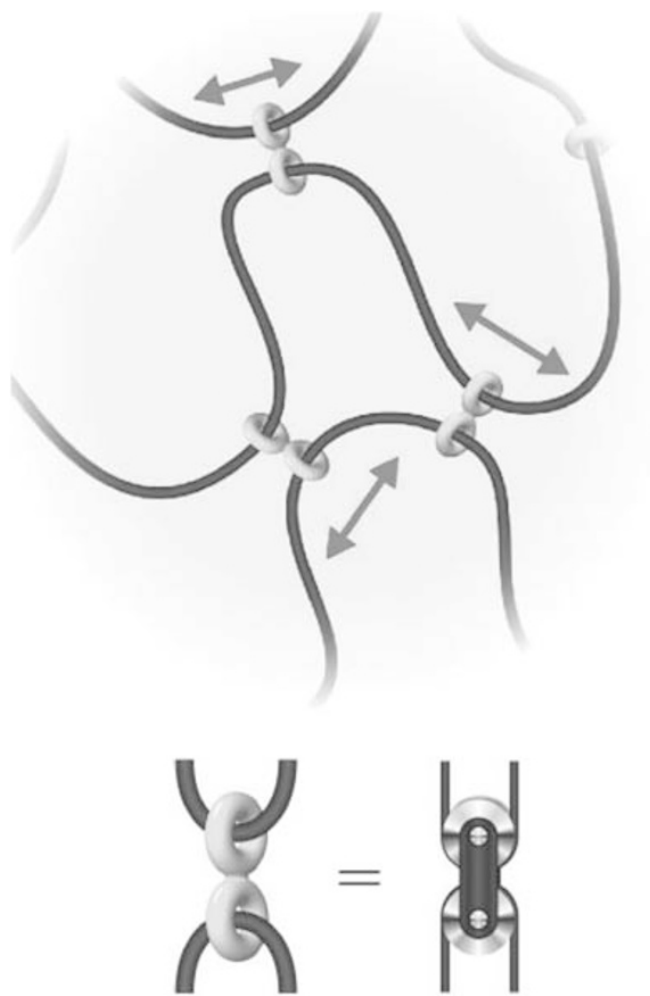

Figure 6 Schematic diagram of slide-ring gels with freely movable figure-eight crosslinks functioning like pulleys. (Reproduced with permission from Ito. ${ }^{31}$ )

\section{Slide-ring gels}

A polymer network in which the polymer chains are tied together by slide-ring pulleys is considered to show unique mechanical properties, as the elasticity of the gel is a consequence of only the chain's topology. Ito and co-workers ${ }^{30-33}$ prepared such a 'slide-ring' (SR) gel and showed its unique properties. SR gels can be prepared from a polyrotaxane architecture consisting of a number of cyclic molecules $(\alpha$-cyclodextrin, $\alpha$-CD) threaded onto a long linear polymer chain (PEG), followed by trapping the cyclic molecules by capping with bulky end groups (Figure 5). Some of the cyclic molecules are then chemically attached to form a figure-eight structure and form the SR gel. The figure-eight crosslinks can move along the long polymer axis to minimize local stress in the gel, similar to pulleys, and this sliding motion gives rise to remarkable physical properties in SR gels (Figure 6). SR gels are considered an intermediate between physical gels and chemical gels, as the polymer network is not formed by either covalent crosslinks, as in chemical gels, or attractive interactions, as in physical gels. This pulley effect has been confirmed through analysis of the static structure of SR gels under uniaxial deformation using smallangle neutron scattering ${ }^{34,35}$ and small-angle X-ray scattering. ${ }^{36} \mathrm{~A}$ prolate pattern perpendicular to the deformation direction, which is known as a normal butterfly pattern or an almost isotropic pattern, has been reported for SR gels. This pattern is evidence of the pulley effect in SR gels and represents the first observation of a normal butterfly pattern in any polymeric gel. The sliding mode of $\alpha$-CD in polyrotaxanes and that of crosslinking junctions in SR gels have also been observed in quasi-elastic light scattering studies ${ }^{37}$ and dynamic light scattering studies. ${ }^{38}$ SR gels have high stretchability, reaching up to 24 times their original length without hysteresis. In the stress-strain

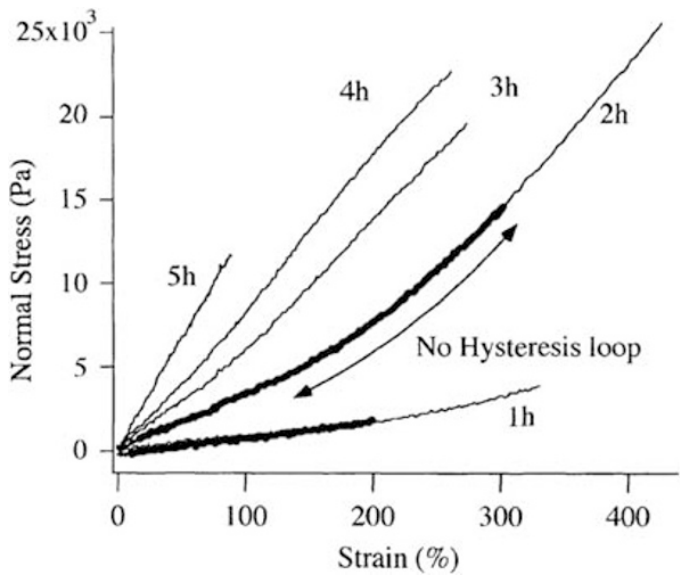

Figure 7 Stress-strain curves of slide-ring gels with different gelation times. Slide-ring gels show non-affine curves without a hysteresis loop. (Reproduced with permission from Okumura and Ito. ${ }^{39}$ )

curve of SR gels, a J-shaped curve without any hysteresis loop has been reported, which is completely different from the stress-strain curves of both physical and chemical gels (Figure 7). ${ }^{39,40}$ The mechanical strength of SR gels has been reported to be similar to that of mammalian skin, vessels and tissues. SR gels become stiffer as the extension ratio reaches the fracture point; therefore, they could be used as a substitute for various types of biomaterials. Koga and Tanaka $^{41}$ reported a molecular simulation of Brownian dynamics using a simple model of a polymer network with trifunctional sliding junctions to study the elastic properties of SR gels compared with conventional polymer gels. SR gels exhibit a huge transient overshoot in the swelling and shrinking process at a stepwise change in solvent from $0.1 \mathrm{M} \mathrm{NaCl}$ aqueous solution to water and from water to acetone, respectively. This overshoot occurs approximately $20 \mathrm{~min}$ after changing the solvent and is assumed to be closely related to the pulley effect. Depending on solvent composition, the volume change of SR gels has been used to prepare stimuli-responsive photonic band gap materials using a closely packed colloidal crystal as a template in which the structural color changes. ${ }^{42}$ Furthermore, the modification of $\alpha-C D$ by some stimuli-sensitive groups can provide SR gels with stimuli sensitivity. For example, a new type of photoresponsive polymer gel has been developed by introducing an azobenzene moiety on the $\alpha-\mathrm{CD}$ of polyrotaxane. ${ }^{43}$ This azobenzene-modified SR gel exhibits enormous photo-induced deformation and characteristic transient overshoot behavior during expansion.

\section{Tetra-PEG gels}

Solving the inherent inhomogeneity of hydrogel network structures is one of the best ways to improve the mechanical properties of hydrogels. A novel hydrogel named a 'tetra-PEG gel' was reported by Sakai et al., ${ }^{44,45}$ in which the combination of two symmetrical tetrahedron-like macromers of the same size resulted in a homogeneous, almost ideal network structure. In that study, four-armed PEG was used to prepare two types of macromers with a tetrahedronlike structure: tetra-amine-terminated PEG and tetra-succinimidyl ester-terminated PEG (Figure 8). Mixing two macromer solutions under controlled $\mathrm{pH}$ forms amide bonds between the terminal amino groups and the succinimidyl ester groups; thus, a highly homogeneous, symmetrical, tough tetra-PEG hydrogel network is formed (Figure 9a). Unlike conventional hydrogels, the formation of topological inhomogeneities such as dangling chains, entanglements and 
a

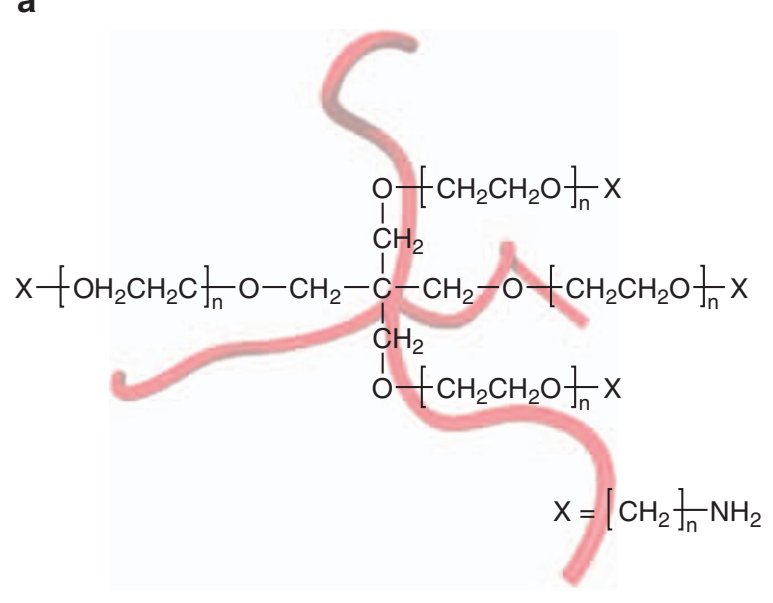

b

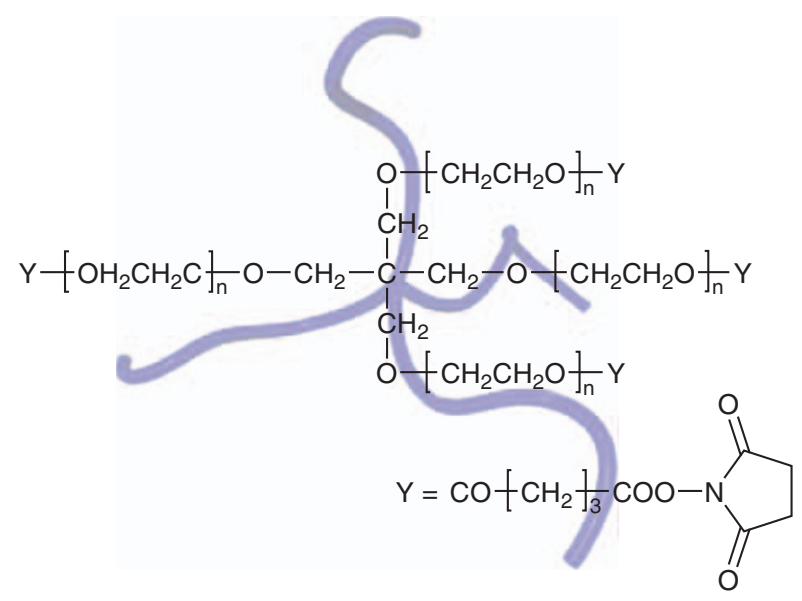

Figure 8 Molecular structures of (a) tetra amine-terminated poly(ethylene glycol) (PEG) and (b) tetra-succinimidyl ester-terminated PEG. (Reproduced with permission from Sakai et al. ${ }^{44}$ )

loops is negligible in tetra-PEG gels. Dynamic light scattering results suggest that tetrahedral macromers connect to each other alternately to form a network with a characteristic length corresponding to a twoarm length. Tetra-PEG gels can be well fitted to available theoretical scattering functions (the Ornstein-Zernike function), and no observable excess scattering appears in the small-angle neutron scattering results. ${ }^{46}$ Young's modulus of tetra-PEG gels is proportionally correlated to polymer volume fractions, indicating the absence of entanglements in the network. ${ }^{47}$ It has been observed that the compressive strength of tetra-PEG gels is on the order of a few to tens of MPa for a $120 \mathrm{mg} \mathrm{ml}^{-1}$ gel made from a macromer with $M_{\mathrm{w}}=10000$. This strength is as high as that of articular cartilage (Figure 9b). The technique to prepare the homogeneous structure of tetra-PEG gels could be used to prepare stimuli-sensitive hydrogels composed of other functional polymers.

\section{Homogenous hydrogels by a click reaction}

Malkoch et al. used click chemistry to prepare a well-defined, PEGbased homogeneous hydrogel network with superior mechanical strength. ${ }^{48,49}$ In that approach, copper (I)-catalyzed 1,2,3-triazoles formed from azides and terminal acetylene were used for a specific click reaction (Figure 10). ${ }^{50}$ The unreacted azide and/or acetylene
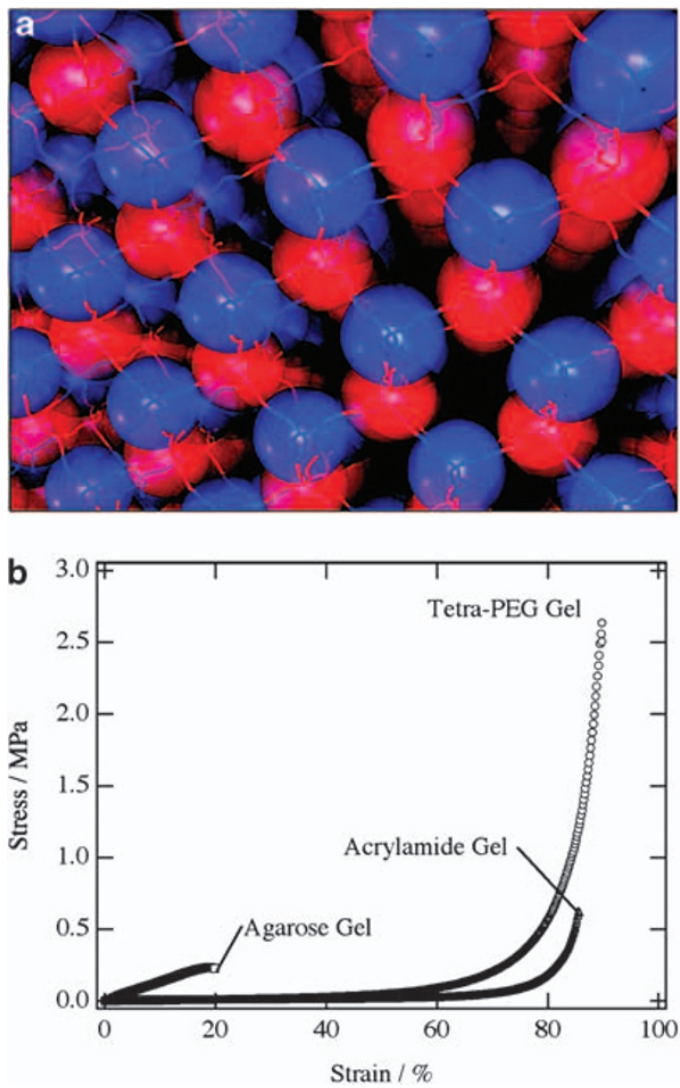

Figure 9 (a) Schematic illustration of a model structure for a tetra-PEG gel formed at $C^{*}$ (the concentration defining the border between dilute and semidilute regions). Red and blue spheres represent tetra amine-terminated poly(ethylene glycol) (PEG) and tetra-succinimidyl ester-terminated PEG, respectively. (b) Stress-strain curves of an agarose gel (square), acrylamide gel (triangle) and tetra-PEG gel (circle). (Reproduced with permission from Sakai et al. ${ }^{44}$ )

groups allow the incorporation of various additives and functional groups in the hydrogel network without affecting network formation, and the gelation can be performed under mild conditions at room temperature. The click technique forms a gel network via controlled crosslinking, providing an ideal gel network and resulting in improved swelling and mechanical behaviors. Hydrogels prepared by a click reaction using long PEG $\left(M_{\mathrm{n}}=10000\right)$ have true stress of $\sim 2390 \mathrm{kPa}$ and elongation at a break of $\sim 1550 \%$. No studies have yet reported the stimuli sensitivity of click gels, but we believe that these gels will exhibit interesting stimuli sensitivity because of their well-defined and homogeneous gel network.

\section{Nanocomposite hydrogels}

Creative crosslinker structures such as those found in SR gels are also a good way to improve the mechanical properties of hydrogels. Haraguchi and Takehisa ${ }^{51}$ used various types of clay particles as crosslinkers to prepare nanocomposite hydrogels (NC gels). They first reported NC gels prepared by in situ free-radical polymerization of NIPA in the presence of uniformly dispersed inorganic exfoliated clay particles (clay platelets) in aqueous media (Figure 11). The clay platelets function as multifunctional crosslinkers: the ends of the polymer chains adsorb strongly on the surface of the clay platelets by ionic and coordination interactions. ${ }^{52}$ The intercrosslinking distance is equivalent to the distance between neighboring clay particles 
<smiles>COCC(C)O</smiles>

1a, $M_{\mathrm{n}}=3400$

1b, $M_{\mathrm{n}}=6000$

1c, $M_{\mathrm{n}}=8000$

1d, $M_{\mathrm{n}}=10000$<smiles>CC1(C)OCC(C)(C(=O)OC(=O)C2(C)COC(C)(C)OC2)CO1</smiles><smiles>CC(COC(=O)C(C)(CO)CO)OC(C)C(=O)C(C)(CO)CO</smiles>

3

1. $\mathrm{MeSO}_{2} \mathrm{Cl}, \mathrm{NEt}_{3}$

2. $\mathrm{NaN}_{3}$<smiles>C#CCCC(=O)OCCOC(=O)CCC#C</smiles>

2a-d

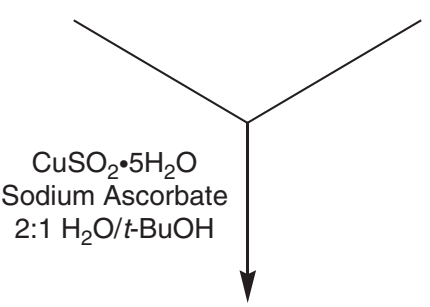<smiles>CC(CN)(CN)C(=O)OCCOC(=O)C(C)(CN)CN</smiles>

4<smiles>CC(C)(C)OCC(C)(C)OC(=O)CCc1cn(CC(C)(Cn2cc(CCC(=O)OC(C)(C)COC(C)(C)C)nn2)C(=O)OCCOC(=O)C(C)(Cn2cc(CCC(=O)OC(C)(C)COC(C)(C)C)nn2)Cn2cc(CCC(=O)OC(C)(C)COC(C)(C)C)nn2)nn1</smiles>

Figure 10 Modular approach for hydrogel construction based on click chemistry and poly(ethylene glycol)-based building blocks. (Reproduced with permission from Malkoch et al. ${ }^{48}$ )

and is very large. In the elongated state of NC gels, these large, flexible polymer chains are capable of being highly and reversibly elongated without breaking the short polymer chains, giving superior mechanical strength to NC gels. NC gels also exhibit high levels of deformation on elongation, compression, tearing, bending, twisting and knotting. ${ }^{53,54}$ Depending on the kind of clay particles and monomer used for NC gels, these gels can generally be elongated to more than $1000 \%$ of their original length (Figure 12). The modulus value and the fracture energy of NC gels are also much higher than those of conventional poly(NIPA) gels. ${ }^{55}$ By increasing concentration of clay platelets $\left(C_{\text {clay }}\right)$ up to $25 \mathrm{~mol} \%$, NC gels exhibit noteworthy increases in strength, modulus and fracture energy without sacrificing elongation at break. ${ }^{56}$ Irrespective of the nature of polymer and clay content, NC gels are generally very transparent, indicating the formation of uniform network structures with fewer spatial inhomogeneities, unlike that found in conventional hydrogels. The static and dynamic structures of NC gels as a function of the $C_{\text {clay }}$ have been reported by studies using small-angle neutron scattering, small-angle X-ray scattering and dynamic light scattering. ${ }^{57-59}$ NC gels using NIPA with small amounts of clay platelets exhibit higher swelling ratios and faster deswelling compared with conventional poly(NIPA) gels. ${ }^{60,61}$ In contrast, with increasing $C_{\text {clay }}$, the mobility of poly(NIPA) chains gradually decreases because of the high crosslinking density; consequently, the deswelling rate also decreases. Moreover, when a large amount of clay is used, the thermosensitivity of NC gels is completely lost. ${ }^{55}$ However, given that NC gels are essentially a kind of physical gel, their network structure can be dissolved in certain environments. Even so, NC gels are very useful for many applications.

\section{Double network gels}

Gong et al. prepared double network (DN) gels, previously known as IPN gels, with superior mechanical strength and toughness. ${ }^{62} \mathrm{DN}$ gels can be synthesized by two kinds of crosslinked polymer networks, in which one network is made from heavily crosslinked rigid poly- 

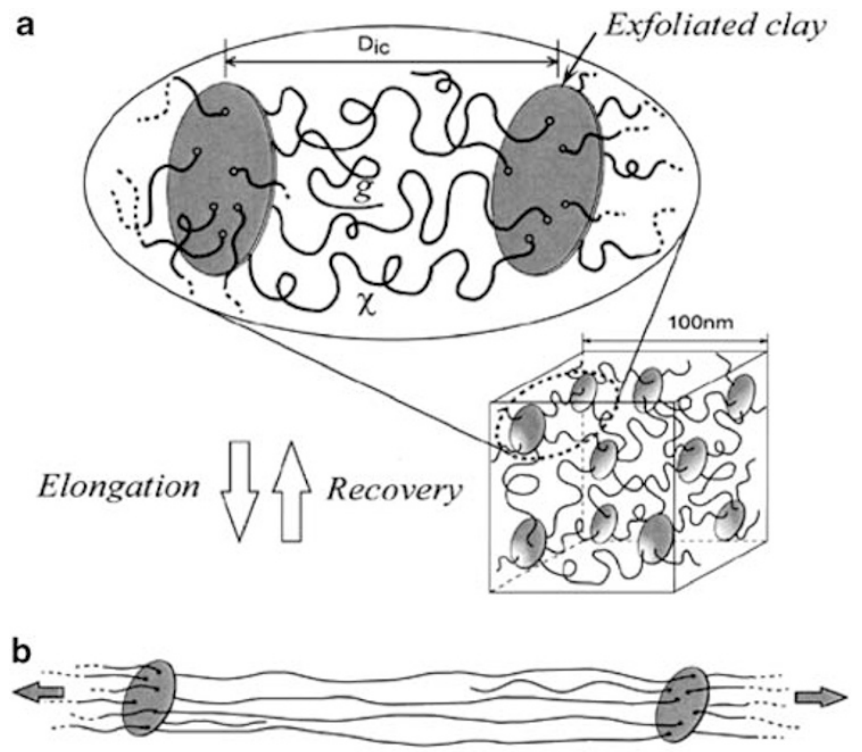

c

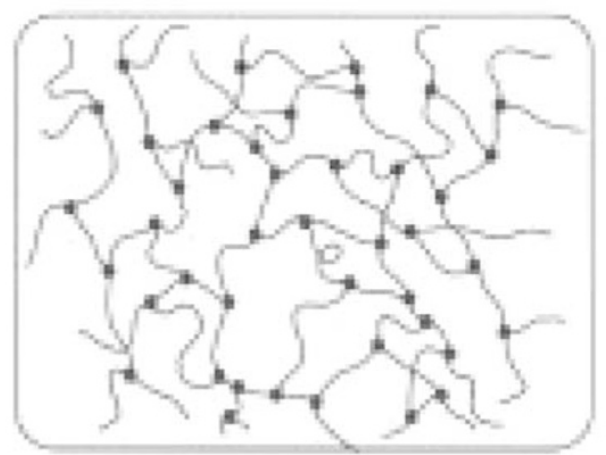

Figure 11 Network structure models for nanocomposite hydrogels (NC gels) and conventional gels. (a) Schematic representation of a 100-nm cube of an NC gel consisting of uniformly dispersed (exfoliated) inorganic clay and two primary types of flexible polymer chains, $\chi$ and $g$, grafted onto two neighboring clay sheets and one clay sheet, respectively. In the model, only a small number of polymer chains are depicted for simplicity. (b) Elongated structure model for NC gels. (c) Conventional gel network structure model. (Reproduced with permission from Haraguchi and Takehisa. ${ }^{51}$ )

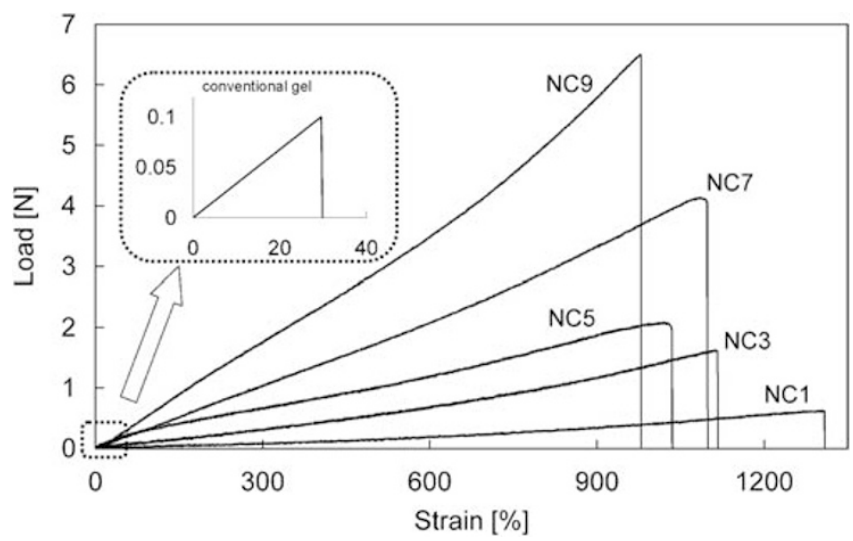

Figure 12 Load-strain curves of nanocomposite hydrogels (NC gels) with different clay contents (increasing clay content from NC1 to NC9). The approximate data for conventional gels are also depicted for comparison. All hydrogels had the same polymer/water ratio $(10: 1(\mathrm{w} / \mathrm{w}))$. (Reproduced with permission from Haraguchi et al. ${ }^{53}$ )

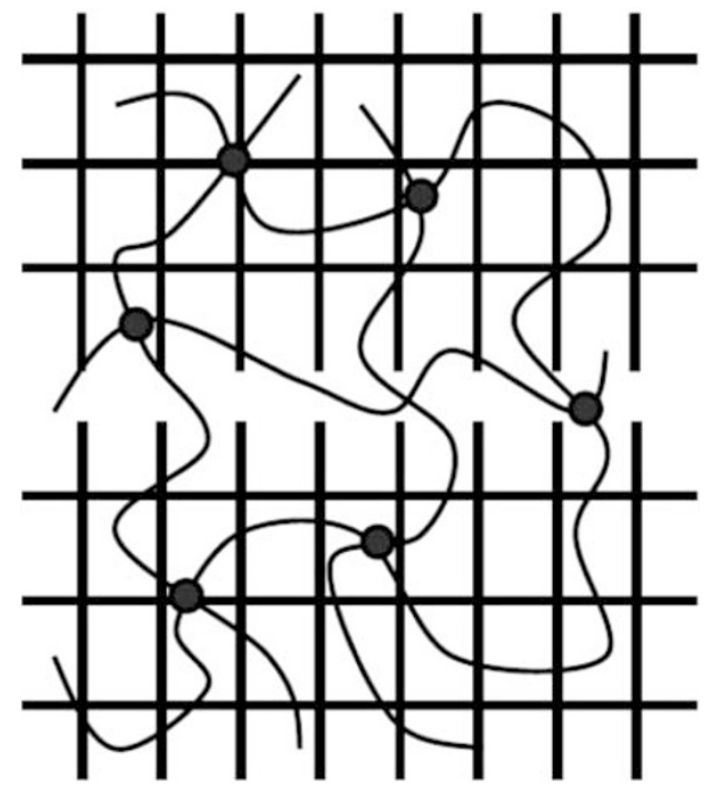

Figure 13 Structural model of a methyl-propane sulfonate polymer/ acrylamide polymer double network gel. (Reproduced with permission from Nakajima et al. ${ }^{66}$ )

electrolyte polymer and the other is made from lightly crosslinked or, in some cases, an uncrosslinked, flexible uncharged polymer (Figure 13). At first, a moderately tight crosslinked gel is formed. After swelling, this first network in a solution of a second monomer with little or no crosslinking agent is used to prepare the second network. Given that the first network is highly swollen in the second monomer solution, the second network is effectively entangled with the first network to form a very strong DN gel. ${ }^{62-67}$ The first network contributes to an increase in elastic stress, whereas the second network contributes to an increase in strain. Numerous polymer pairs have been used to optimize the characteristic behaviors of DN gels, and the methyl-propane sulfonate polymer/acrylamide polymer pair has been found to be the most promising. Methyl-propane sulfonate polymer/ acrylamide polymer-based DN gels exhibit both hardness (elastic modulus of $0.3 \mathrm{MPa}$ ) and toughness (compressive strength of $\sim 20 \mathrm{MPa}$ ) (Figure 14). The tearing fracture energy of methyl-propane sulfonate polymer/acrylamide polymer-based DN gels under optimized conditions can be as high as $10^{3} \mathrm{Jm}^{-2}$, much higher than that of acrylamide polymer $\left(\sim 10 \mathrm{Jm}^{-2}\right)$ and methyl-propane sulfonate polymer gels $\left(\sim 0.1 \mathrm{Jm}^{-2}\right)$ alone. ${ }^{68,69}$

The structure and property relationships of DN gels have been studied by small-angle neutron scattering, ${ }^{70}$ dynamic light scattering, ${ }^{71}$ molecular dynamics simulations, ${ }^{72,73}$ modeling of crack formation $^{74}$ and void formation. ${ }^{71}$ Entanglements between two polymer networks are a prerequisite condition in all cases for the toughness of DN gels. ${ }^{75}$ After the innovation of DN gels, some research groups began studying various avenues of research with $\mathrm{DN}$ gels, but no detailed observations of stimuli sensitivity have been reported thus far, except in the work of Liang et al. ${ }^{76}$ Recently, they reported a fast and high solvent-triggered force generation considering their mechanical toughness for very thin DN gels. As high-level inhomogeneities are the origin of superior toughness of DN gels, the stimuli sensitivity of bulk DN gels could be very complex. Moreover, in such IPNs, the stimuli sensitivities for each polymer become seriously impaired because of interactions between the polymers. Thus, it might be considerably 
difficult to design a stimuli-sensitive hydrogel with good mechanical properties simply using a DN gel. As the mechanical properties of DN gels are substantially superior, however, these gels should have broad applications.

\section{OUR APPROACH FOR MAKING HYDROGELS WITH FAST STIMULI SENSITIVITY AND SUPERIOR MECHANICAL PERFORMANCE}

Here, we report a facile and universal approach to introduce the slidering phenomenon into conventional hydrogels in order to obtain

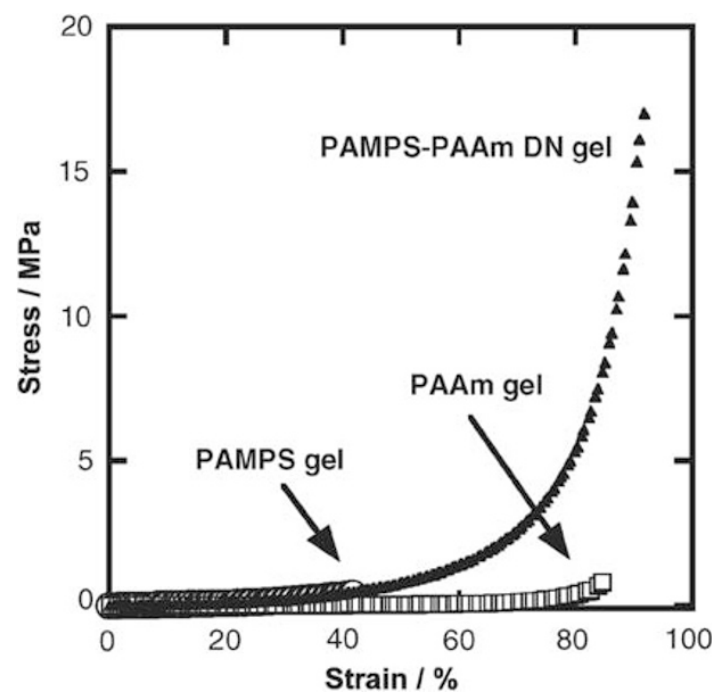

Figure 14 Stress-strain curves for hydrogels under uniaxial compression. Circles: methyl-propane sulfonate polymer gel (water content: $90 \mathrm{wt} \%$ ); squares: acrylamide polymer gel (water content: 90 wt\%); triangles: methylpropane sulfonate polymer-acrylamide polymer double network gel (water content: 90 wt\%). (Reproduced with permission from Gong et al. ${ }^{62}$ ) highly mechanically stable and fast stimuli-responsive hydrogels. We focused on the exploitation of the intriguing properties of polyrotaxanes as crosslinkers in the synthesis of stimuli-sensitive hydrogels. A sparsely dispersed polyrotaxane was used, consisting of larger numbers of $\alpha$-CD and long PEG $\left(M_{\mathrm{w}}=35000\right)$ molecules in the presence of bulky-end 1-adamantanamine groups in the polymer axis, which prevented the dethreading of $\alpha-\mathrm{CD}$. The hydroxyl groups of $\alpha$-CD or $\alpha$-CD derivatives of polyrotaxanes were modified by isocyanate monomer (2-acryloyloxyethylisocyanate) through the formation of a stable carbamate bond, resulting in the hydrophobic polyrotaxane crosslinker, MPR and hydrophilic polyrotaxane crosslinker, MHPR, respectively. ${ }^{77-79}$ The solubility and degree of substitution (DS) $(0 \leq \mathrm{DS} \leq 18)$, that is, the average number of substituted hydroxyl groups per $\alpha$-CD molecule, could be tuned by changing the derivatives of $\alpha$-CD or the amount of isocyanate monomer. Hydrogels were fabricated using conventional free-radical polymerization of thermosensitive NIPA monomer or using several types of ionic comonomers in the presence of MPRs or MHPRs as crosslinkers (Figure 15). As a result, we obtained mechanically superior poly (NIPA) hydrogels: rotaxane-NIPA (RN) gels or ionic rotaxane-NIPA (iRN) gels. Moreover, these new types of crosslinkers could easily be applied to a wide range of monomers to synthesize hydrogels by freeradical polymerization and improve their stimuli responsiveness and mechanical properties. RN gels exhibit irregular swelling behavior and resemble graft or block copolymer gels in that the volume phase transition temperature of $\mathrm{RN}$ gels is similar to that of conventional poly(NIPA) hydrogels.

Depending on the amount of hydrophilic crosslinker, MHPR, higher swelling ratios have been reported for RN gels. ${ }^{78,79}$

In water, RN gels exhibit a sharp volume phase change around the LCST of poly(NIPA). After a sudden temperature jump, RN gels shrink isotropically without creating any deformation on their surface, and the rate of deswelling is very fast. Two stages of relaxation of the volume change have been observed in $\mathrm{RN}$ gels during the shrinking process, but most of the change occurs in the first stage. The first-stage

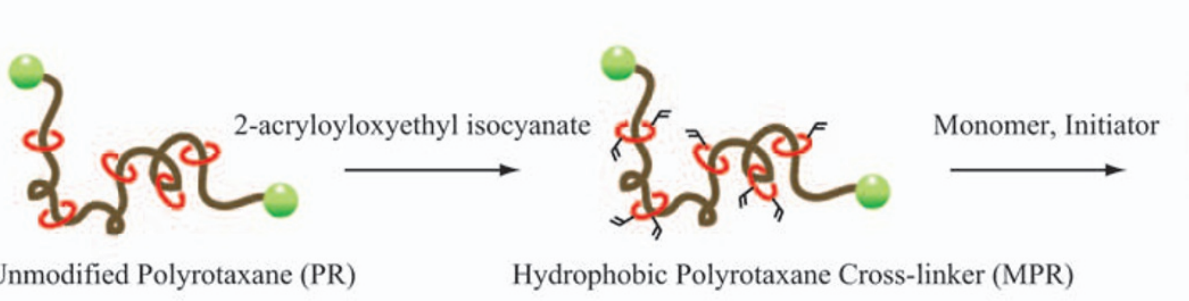

Unmodified Polyrotaxane (PR)

Hydrophobic Polyrotaxane Cross-linker (MPR)

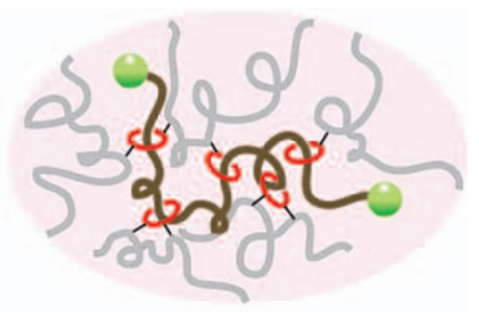

RwiN gel

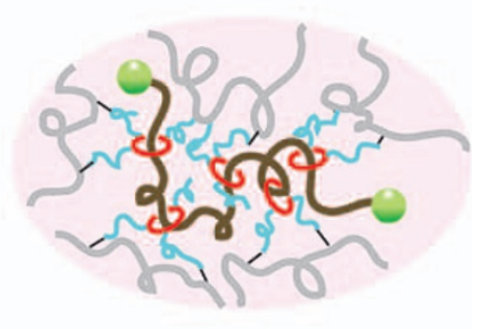

RwsN gel

Figure 15 Schematic representation of the preparation of hydrophobic and hydrophilic polyrotaxane crosslinkers (MPR and MHPR) and their applications to the preparation of rotaxane-( $N$-isopropylacrylamide) gels in dimethylsulfoxide. (Reproduced with permission from Bin Imran et al. ${ }^{78}$ ) 


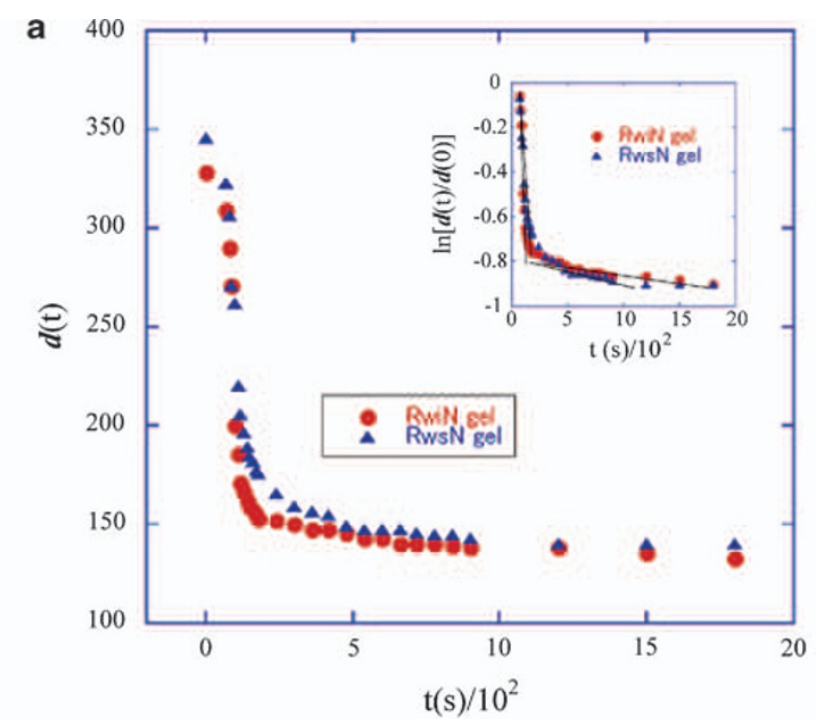

b $\mathrm{R}_{\mathrm{ws}} \mathrm{N}$ gel

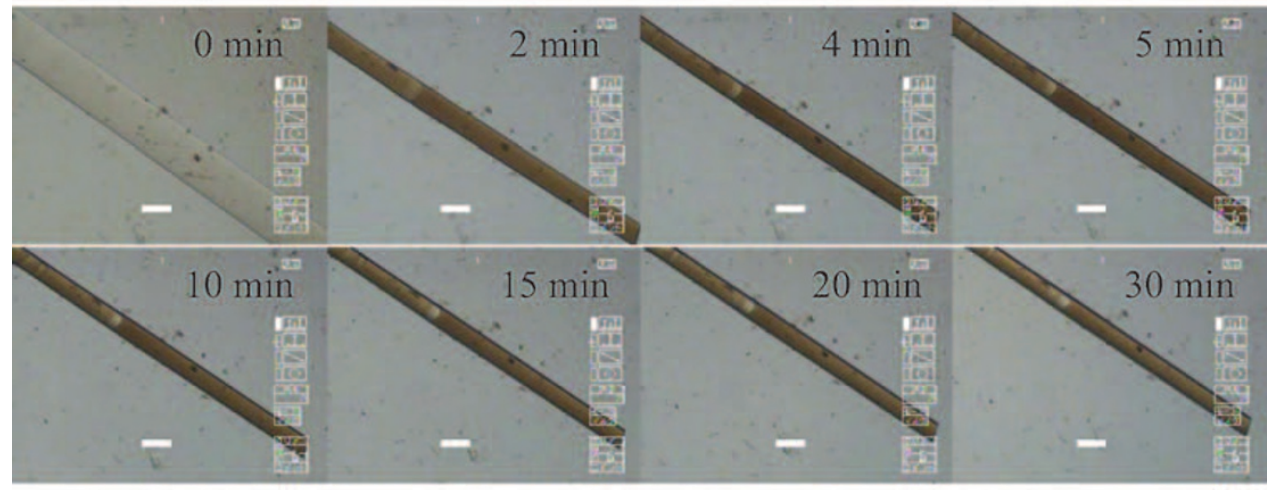

$\mathrm{R}_{\mathrm{ws}} \mathrm{N}$ gel

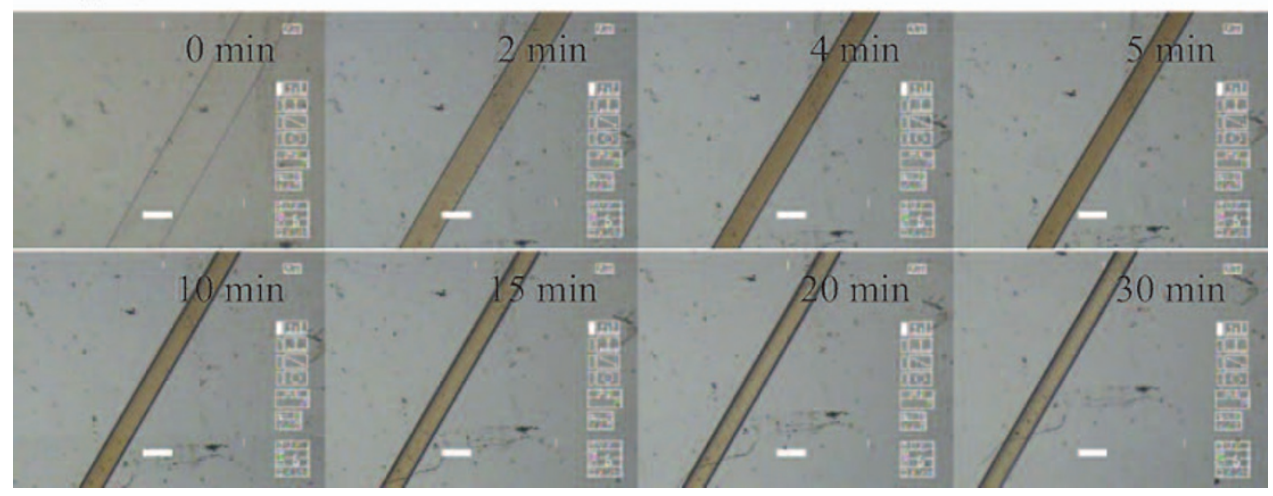

Figure 16 (a) Fast-shrinking kinetics of cylindrical $R_{w i} N$ and $R_{w s} N$ hydrogels after a temperature jump from 20 to $40{ }^{\circ} \mathrm{C}$. Inset in part a): plots of In[d(t)/d(0)] versus time to calculate the relaxation times at different stages of rotaxane-( $N$-isopropylacrylamide) (RN) gels. The relaxation times were determined from the slopes $(-1 / \tau)$ of each straight line at different stages. (b) Micrographs of morphological changes of cylindrical RN gels in water after a sudden temperature jump from 20 to $40^{\circ} \mathrm{C}$. Scale bar: $200 \mu \mathrm{m}$. (Reproduced with permission from Bin Imran et al. ${ }^{78}$ )

relaxation time $\tau_{1}$ for $\mathrm{R}_{\mathrm{wi}} \mathrm{N}$ gels (MPR-based gels) and $\mathrm{R}_{\mathrm{ws}} \mathrm{N}$ (MHPRbased gels) gels is $1.26 \times 10^{2}$ and $2.11 \times 10^{2} \mathrm{~s}$, respectively, whereas the second-stage relaxation time $\tau_{2}$ is $1.27 \times 10^{4}$ and $6.1 \times 10^{3} \mathrm{~s}$, respectively (Figure 16). ${ }^{78}$ The second-stage relaxation for RN gels might be derived from the final rearrangement of aggregated nuclei to reach equilibrium-shrunken states. Above the LCST, the crosslinked poly (NIPA) chains of conventional poly(NIPA) gels form a phase-sepa- rated insoluble globular structure and the fixed crosslinking facilitates the formation of an insoluble skin layer on the surface. Water molecules cannot pass through this impermeable skin layer. On the other hand, the dynamic nature of the crosslink points may help to aggregate polymer networks to reach the complete collapsed state before forming any impermeable skin layer on the surfaces of RN gels. The micro- and macro-level spatial inhomogeneities and local stresses 
inside the polymer networks of RN gels can automatically be homogenized and relaxed by movement of the mobile crosslink points. Thus, RN gels shrink by the growth of nucleation without going through the unstable spinodal decomposition mechanism, resulting in very fast shrinking rates. Although $\mathrm{R}_{\mathrm{wi}} \mathrm{N}$ gels reach an equilibriumshrunken state very quickly and isotropically, hydrophobic aggregation of the water-insoluble MPR gives the gel permanent opacity in the collapsed state. To overcome this problem, the hydrophilic polyrotaxane crosslinker MHPR has been used to prepare $\mathrm{R}_{\mathrm{ws}} \mathrm{N}$ gels. The hydrophilicity of the crosslinker retains the homogeneity of the $\mathrm{R}_{\mathrm{ws}} \mathrm{N}$ gel network and restricts the formation of aggregated globules, permitting the poly(NIPA) chains inside the gel network to move and rotate freely along the long polymer axis under deformation. RN gels exhibit much smaller storage moduli $E^{\prime}$ and loss moduli $E^{\prime \prime}$ than do conventional poly(NIPA) hydrogels in the frequency ranges studied, indicating the very soft nature of RN gels. When a frequency is applied to RN gels, the poly(NIPA) chains inside the gel network squeeze enough. Because of the sliding and rotating ability of the crosslinks in the RN gels, however, the poly(NIPA) chains can gradually equalize this tension. These movable crosslinks allow the poly(NIPA) chains to relax under deformation, whereas the fixed crosslink networks of conventional poly(NIPA) hydrogels cannot avoid localization of the stress to short poly(NIPA) chains and soon lose their mechanical integrity. An increase in the amount of crosslinker or temperature results in increases in both $E^{\prime}$ and $E^{\prime \prime}$ in both water and dimethylsulfoxide. Thus, the softness or hardness of RN gels seems to be well controlled. Although RN gels prepared by hydrophobic and hydrophilic polyrotaxane crosslinkers show significant improvements in various respects, they do not show appreciable tensile strength under deformation. The shrinkage of the crosslinkers in water may obstruct the fluid movement of $\alpha-C D$, which could prevent improvement of the mechanical properties of RN gels.

When a small amount of ionic comonomer (for example, sodium acrylate) is introduced into RN gel networks, the electrostatic repulsion between the ionic groups and the osmotic pressure due to the presence of the counter ions increases the dispersity of the polymer chains attached to $\alpha$-CD through the long PEG chains, thereby, enhancing the mechanical strength of the hydrogel (A Bin Imran et al., in preparation). The discontinuous volume changes of iRN gels with temperatures could be changed to continuous ones only by changing the $\mathrm{pH}$ of the aqueous medium from acidic to basic. iRN gels show normal butterfly patterns in their small-angle X-ray scattering profiles, strongly suggesting the presence of sliding crosslinks that effectively reduce spatial inhomogeneities. The polymer chains in conventional hydrogels are stretched and organized in the stretching, and the frozen structures are displaced along the stretching direction without any notable changes in shape, indicating that spatial inhomogeneity increases in the stretching direction and gives an abnormal butterfly pattern in the scattering pattern. In contrast, the spatial inhomogeneities of iRN gels do not increase in the stretching direction because of the presence of movable crosslinks; consequently, normal butterfly patterns appear in the scattering profile. iRN gels exhibit high levels of deformation with elongation, compression, tearing, bending and twisting and do not readily tear at any cut or notch. The elongation at the break of iRN gels is very high $(\sim 1000 \%$ strain$)$ and decreases with increasing amounts of crosslinker. Interestingly, the tensile strength of iRN gels is similar to that of SR gels. The high mechanical integrity of iRN gels suggests that iRN gels could potentially be used as biomaterials, as the softness of the gels would reduce the mechanical and frictional irritation to surrounding tissues. The phenomena observed in iRN gels could successfully be exploited for the development of materials with diverse applications, such as photosensitive materials, biomedical applications, drug delivery systems, smart actuators, contact lenses, artificial cartilage/joints, artificial blood vessels, wound healing materials, shock-absorption materials and adhesives.

\section{CONCLUSIONS}

The techniques described in this review are very promising for improvement of the stimuli sensitivity and mechanical properties of hydrogels. If a hydrogel can be developed that combines high-quality mechanical performance with fast stimuli responsiveness, it could be widely used for applications ranging from biomedical to commercial. Advanced techniques to improve the mechanical properties and fast stimuli sensitivity of hydrogels simultaneously are expected to expand the range of applications for hydrogels. Among all the hydrogels described, only NC gels and RN gels have been reported to have both fast deswelling kinetics and superior mechanical strength. Research in this area is still in its rudimentary stage and must go further to significantly improve the applications of hydrogels in diverse areas.

\section{ACKNOWLEDGEMENTS}

This work was partially supported by a Grant-in-Aid for Scientific Research on Innovative Area of 'Fusion Materials: Creative Development of Materials and Exploration of Their Function through Molecular Control' (no. 2206) from the Ministry of Education, Culture, Sports, Science, and Technology.

1 Bhattacharya, S., Eckert, F., Boyko, V. \& Pich, A. Temperature-, pH-, and magneticfield-sensitive hybrid microgels. Small 3, 650-657 (2007).

2 Schild, H. G. Poly( $N$-isopropylacrylamide): experiment, theory and application. Prog. Polym. Sci. 17, 163-249 (1992).

3 Peppas, N. A., Hilt, J. Z., Khademhosseini, A. \& Langer, R. Hydrogels in biology and medicine: from fundamentals to bionanotechnology. Adv. Mater. 18, 1345-1360 (2006).

4 Chaterji, S., Kwon, I. K. \& Park, K. Hydrogels for medical and biomedical applications. Prog. Polym. Sci. 32, 1083-1122 (2007).

5 Okano, T., Bae, Y. H., Jacobs, H. \& Kim, S. W. Thermally on-off switching polymers for drug permeation and release. J. Control. Rel. 11, 255-265 (1990).

6 Tanaka, T. \& Fillmore, D. J. Kinetics of swelling of gels. J. Chem. Phys. 70, 1214-1218 (1979).

7 Shibayama, M. \& Tanaka, T. Volume phase transition and related phenomena of polymer gels responsive gels: volume transitions I. Adv. Polym. Sci. 109, 1-62 (1993).

8 Li, Y. \& Tanaka, T. Phase transitions of gels. Annu. Rev. Mater. Sci. 22, 243-277 (1992).

9 Li, Y. \& Tanaka, T. Kinetics of swelling and shrinking of gels. J. Chem. Phys. 92, 13651371 (1990).

10 Hirose, H. \& Shibayama, M. Kinetics of volume phase transition in poly $(\mathrm{N}$-isopropylacrylamide-co-acrylic acid) gels. Macromolecules 31, 5336-5342 (1998).

11 Kabra, B. G. \& Gehrke, S. H. Synthesis of fast response poly( $N$-isopropylacrylamide) gels. Polym. Commun. 32, 322-323 (1991).

12 Zhang, X. Z., Yang, Y. Y. \& Chung, T. S. Effect of mixed solvents on characteristics of poly( $N$-isopropylacrylamide) gels. Langmuir 18, 2538-2542 (2002).

13 Oxley, H. R., Corkhill, P. H., Fitton, J. H. \& Tighe, B. J. Macroporous hydrogels for biomedical applications: methodology and morphology. Biomaterials 14, 1064-1072 (1993).

14 Cheng, S. X., Zhang, J. T. \& Zhuo, R. X. Macroporous poly( $N$-isopropylacrylamide) hydrogels with fast response rates and improved protein release properties. J. Biomed. Mater. Res. 67A, 96-103 (2003).

15 Takeoka, Y. \& Watanabe, M. Polymer gels that memorize structures of mesoscopically sized templates. Langmuir 18, 5977-5980 (2002).

16 Zhang, X. Z., Wang, F. J. \& Chu, C. C. Thermoresponsive hydrogel with rapid response dynamics. J. Mater. Sci.: Mater. Med. 14, 451-455 (2003).

17 Zhang, X. Z. \& Zhuo, R. X. Preparation of fast responsive, temperature-sensitive poly ( $n$ isopropylacrylamide) hydrogel. Macromol. Chem. Phys. 200, 2602-2605 (1999).

18 Xue, W., Hamley, I. W. \& Huglin, M. B. Rapid swelling and deswelling of thermoreversible hydrophobically modified poly ( $n$-isopropylacrylamide) hydrogels prepared by freezing polymerization. Polymer 43, 5181-5186 (2002).

19 Yoshida, R., Uchida, K., Kaneko, Y., Sakai, K., Kikuchi, A., Sakurai, A. \& Okano, T. Comb-type grafted hydrogels with rapid de-swelling response to temperature changes. Nature 374, 240-242 (1995). 
20 Kaneko, Y., Sakai, K., Kikuchi, A., Yoshida, R., Sakurai, Y. \& Okano, T. Influence of freely mobile grafted chain length on dynamic properties of comb-type grafted poly (n-isopropylacrylamide) hydrogels. Macromolecules 28, 7717-7723 (1995).

21 Liu, Q. F., Zhang, P. \& Lu, M. G. Synthesis and swelling behavior of comb-type grafted hydrogels by reversible addition-fragmentation chain transfer polymerization. J. Polym. Sci., Part A: Polym. Chem. 43, 2615-2624 (2005).

22 Liu, Q. F., Zhang, P., Qing, A. X., Lan, Y. X. \& Lu, M. G. Poly(n-isopropylacrylamide) hydrogels with improved shrinking kinetics by RAFT polymerization. Polymer 47, 2330-2336 (2006).

23 Noguchi, Y., Okeyoshi, K. \& Yoshida, R. Design of surfactant-grafted hydrogels with fast response to temperature. Macromol. Rapid Commun. 26, 1913-1917 (2005).

24 Xu, X. D., Zhang, X. Z., Yang, J., Cheng, S. X., Zhuo, R. X. \& Huang, Y. C. Strategy to introduce a pendent micellar structure into poly( $\mathrm{N}$-isopropylacrylamide) hydrogels. Langmuir 23, 4231-4236 (2007).

25 Gil, E. S. \& Hudson, S. M. Effect of silk fibroin interpenetrating networks on swelling/ deswelling kinetics and rheological properties of poly $(\mathrm{N}$-isopropylacrylamide) hydrogels. Biomacromolecules 8, 258-264 (2007).

26 Zhang, X. Z., Wu, D. Q. \& Chu, C. C. Synthesis, characterization and controlled drug release of the thermosensitive IPN-PNIPAAm hydrogels. Biomaterials 25, 3793-3805 (2004).

27 Asoh, T.- A., Kaneko, T., Matsusaki, M. \& Akashi, M. Rapid deswelling of semi-IPNs with nanosized tracts in response to $\mathrm{pH}$ and temperature. J. Control. Rel. 110, 387-394 (2006).

28 Ebara, M., Aoyagi, T., Sakai, K. \& Okano, T. Incorporation of carboxylate groups into temperature-responsive poly( $N$-Isopropylacrylamide)-based hydrogels promotes rapid gel shrinking. J. Polym. Sci., Part A: Polym. Chem. 39, 335-342 (2001).

29 Hirotsu, S. Coexistence of phases and the nature of first-order phase transition in Poly$N$-isopropylacrylamide gels. Adv. Polym. Sci. 110, 1-26 (1993).

30 Okumura, Y. \& Ito, K. The polyrotaxane gel: a topological gel by figure-of-eight crosslinks. Adv. Mater. 13, 485-487 (2001).

31 Ito, K. Novel cross-linking concept of polymer network: synthesis, structure, and properties of slide-ring gels with freely movable junctions. Polym. J. 39, 489-499 (2007).

32 Ito, K. Slide-ring materials using topological supramolecular architecture. Curr. Opin. Solid State Mater. Sci. 14, 28-34 (2010).

33 Araki, J., Zhao, C. \& Ito, K. Efficient production of polyrotaxanes from $\alpha$-cyclodextrin and poly(ethylene glycol). Macromolecules 38, 7524-7527 (2005).

34 Karino, T., Okumura, Y., Ito, K. \& Shibayama, M. SANS studies on spatial inhomogeneities of slide-ring gels. Macromolecules 37, 6177-6182 (2004).

35 Karino, T., Okumura, Y., Zhao, C. M., Kataoka, T., Ito, K. \& Shibayama, M. SANS studies on deformation mechanism of slide-ring gel. Macromolecules 38, 6161-6167 (2005).

36 Shinohara, Y., Kayashima, K., Okumura, Y., Zhao, C., Ito, K. \& Amemiya, Y. Small-angle X-Ray scattering study of the pulley effect of slide-ring gels. Macromolecules 39, 7386-7391 (2005)

37 Zhao, C. M., Domon, Y., Okumura, Y., Okabe, S., Shibayama, M. \& Ito, K. Sliding mode of cyclodextrin in polyrotaxane and slide-ring gel. J. Phys.: Condens. Matter. 17, S2841-S2846 (2005).

38 Mayumi, K. \& Ito, K. Structure and dynamics of polyrotaxane and slide-ring materials. Polymer 51, 959-967 (2010).

39 Okumura, Y. \& Ito, K. The third gel consisting of linear polymer and figure of eight crosslinking points [topological gel] (in Japanese). Nippon Gomu Kyokaishi, 76, 21-26 (2003).

40 Ito, K Proc. of Int. Symp. On Forefront of Nonlinear Science and Its Application to Material Science in the 21st Century, Kyoto, Sept. 28-30 (2005).

41 Koga, T. \& Tanaka, F. Elastic properties of polymer networks with sliding junctions. Eur. Phys. J. E, 17, 225-229 (2005).

42 Murayama, H., Bin Imran, A., Nagano, S., Seki, T., Kidowaki, M., Ito, K. \& Takeoka, Y. Chromic slide-ring gel based on reflection from photonic bandgap. Macromolecules 41, 1808-1814 (2008).

43 Sakai, T., Murayama, H., Nagano, S., Takeoka, Y., Kidowaki, M., Ito, K. \& Seki, T. Photoresponsive slide-ring gel. Adv. Mater. 19, 2023-2025 (2007).

44 Sakai, T., Matsunaga, T., Yamamoto, Y., Ito, C., Yoshida, R., Suzuki, S., Sasaki, N., Shibayama, M. \& Chung, U. I. Design and fabrication of a high-strength hydrogel with ideally homogeneous network structure from tetrahedron-like macromonomers. Macromolecules 41, 5379-5384 (2008).

45 Akagi, Y., Matsunaga, T., Shibayama, M., Chung, U. \& Sakai, T. Evaluation of topological defects in tetra-PEG gels. Macromolecules 43, 488-493 (2010).

46 Matsunaga, T., Sakai, T., Akagi, Y., Chung, U. I. \& Shibayama, M. SANS and SLS studies on tetra-arm peg gels in as-prepared and swollen states. Macromolecules 42 , 6245-6252 (2009).

47 Kurakazu, M., Katashima, T., Chijiishi, M., Nishi, K., Akagi, Y., Matsunaga, T., Shibayama, M., Chung, U. \& Sakai, T. Evaluation of gelation kinetics of tetra-PEG gel. Macromolecules 43, 3935-3940 (2010).

48 Malkoch, M, Vestberg, R., Gupta, N, Mespouille, L, Dubois, P, Mason, A. F., Hedrick, J. L., Liao, Q, Frank, C. W., Kingsbury, K. \& Hawker, C. J. Synthesis of well-defined hydrogel networks using click chemistry. Chem. Commun. 26, 2774-2776 (2006).

49 Polizzotti, B. D., Fairbanks, B. D. \& Anseth, K. S. Three-dimensional biochemical patterning of click-based composite hydrogels via thiolenephotopolymerization. Biomacromolecules 9, 1084-1087 (2008).

50 Rostovtsev, V. V., Green, L. G., Fokin, V. V. \& Sharpless, K. B. A Stepwise Huisgen cycloaddition process: copper(I)-catalyzed regioselective ligation of azides and terminal alkynes. Angew. Chem., Int. Ed. 41, 2596-2599 (2002).
51 Haraguchi, K. \& Takehisa, T. Nanocomposite hydrogels: a unique organic-inorganic network structure with extraordinary mechanical, optical, and swelling/de-swelling properties. Adv. Mater. 14, 1120-1124 (2002)

52 Haraguchi, K., Li, H. J., Matsuda, K., Takehisa, T. \& Elliott, E. Mechanism of forming organic/inorganic network structures during in-situ free-radical polymerization in PNIPA-clay nanocomposite hydrogels. Macromolecules 38, 3482-3490 (2005).

53 Haraguchi, K., Takehisa, T. \& Fan, S. Effects of clay content on the properties of nanocomposite hydrogels composed of poly( $\mathrm{N}$-isopropylacrylamide) and clay. Macromolecules 35, 10162-10171 (2002).

54 Haraguchi, K., Ebato, M. \& Takehisa, T. Polymer-clay nanocomposites exhibiting abnormal necking phenomena accompanied by extremely large reversible elongations and excellent transparency. Adv. Mater. 18, 2250-2254 (2006).

55 Haraguchi, K. \& Li, H. J. Control of the coil-to-globule transition and ultrahigh mechanical properties of PNIPA in nanocomposite hydrogels. Angewandte ChemieInter. Edn. 44, 6500-6504 (2005).

56 Haraguchi, K. \& Li, H. J. Mechanical properties and structure of polymer-clay nanocomposite gels with high clay content. Macromolecules 39, 1898-1905 (2006).

57 Shibayama, M., Karino, T., Miyazaki, S., Okabe, S., Takehisa, T. \& Haraguchi, K. Smallangle neutron scattering study on uniaxially-stretched poly( $N$-isopropylacrylamide)-clay nanocomposite gels. Macromolecules 38, 10772-10781 (2005).

58 Endo, H., Miyazaki, S., Haraguchi, K. \& Shibayama, M. Structure of nanocomposite hydrogel investigated by means of contrast variation small-angle neutron scattering. Macromolecules 41, 5406-5411 (2008).

59 Shibayama, M., Suda, J., Karino, T., Okabe, S., Takehisa, T. \& Haraguchi, K. Structure and dynamics of poly( $\mathrm{N}$-isopropylacrylamide)-clay nanocomposite gel. Macromolecules 37, 9606-9612 (2004).

60 Can, V., Abdurrahmanoglu, S. \& Okay, O. Unusual swelling behavior of polymer- clay nanocomposite hydrogels. Polymer 48, 5016-5023 (2007).

61 Liang, L., Liu, J. \& Gong, X. Thermosensitive poly( $N$-Isopropylacrylamide)-clay nanocomposites with enhanced temperature response. Langmuir 16, 9895-9899 (2000).

62 Gong, J. P., Katsuyama, Y., Kurokawa, T. \& Osada, Y. Double network hydrogels with extremely high mechanical strength. Adv. Mater. 15, 1155-1158 (2003).

63 Nakajima, T., Kurokawa, T., Furukawa, H., Yu, Q. M., Tanaka, Y., Osada, Y. \& Gong, J. P. Super tough gels with a double network structure. Chn. J. Polym. Sci. 27, 1-9 (2009).

64 Tominaga, T., Tirumala, V. R., Lee, S., Lin, E. K., Gong, J. P. \& Wu, W. L. Thermodynamic interactions in double-network hydrogels. J.Phys. Chem. B. 112, 3903-3909 (2008).

65 Tominaga, T., Kurokawa, T., Furukawa, H., Osada, Y. \& Gong, J. P. Friction of a soft hydrogel on rough solid substrates. Soft. Matter. 4, 1645-1652 (2008).

66 Nakajima, T., Furukawa, H., Tanaka, Y., Kurokawa, T., Osada, Y. \& Gong, J. P. True chemical nature of double network hydrogels. Macromolecules 42, 2184-2189 (2009).

67 Dai, T., Qing, X., Lu, Y. \& Xia, Y. Conducting hydrogels with enhanced mechanical strength. Polymer 50, 5236-5241 (2009).

68 Tanaka, Y., Kuwabara, R., Na, Y. H., Kurokawa, T., Gong, J. P. \& Osada, Y. Determination of fracture energy of high strength double network hydrogels. J. Phys. Chem. B. 109, 11559-11562 (2005).

69 Tanaka, Y., Gong, J. P. \& Osada, Y. Novel hydrogels with excellent mechanical performance. Prog. Polym. Sci. 30, 1-9 (2005).

70 Tominaga, T., Tirumala, V. R., Lin, E. K., Gong, J. P., Furukawa, H., Osada, Y. \& Wu, W. The molecular origin of enhanced toughness in double network hydrogels: a neutron scattering study. Polymer 48, 7449-7454 (2007).

$71 \mathrm{Na}$, Y. H., Kurokawa, T., Katsuyama, Y., Tsukeshiba, H., Gong, J. P., Osada, Y., Okabe, S., Karino, T. \& Shibayama, M. Structural characteristics of double network gels with extremely high mechanical strength. Macromolecules 37, 5370-5374 (2004).

72 Jang, S. S., Goddard III, W. A., Yashaar, M., Kalani, S., Myung, D. \& Frank, C. W. Mechanical and transport properties of the poly(ethylene oxide)-poly(acrylic acid) double network hydrogel from molecular dynamic simulations. J. Phys. Chem. B. $111,1729-1737$ (2007)

73 Jang, S. S., Goddard III, W. A., Yashaar, M., Kalani, S., Myung, D. \& Frank, C. W. Corrections to mechanical and transport properties of the poly(ethylene oxide)-poly (acrylic acid) double network hydrogel from molecular dynamic simulations. J. Phys. Chem. B. 111, 14440-14441 (2007).

74 Brown, H. R. A model of the fracture of double network gels. Macromolecules 40, 3815-3818 (2007).

75 Tsukeshiba, H., Huang, M., Na, Y- H., Kurokawa, T., Kuwabara, R., Tanaka, Y., Furukawa, H., Osada, Y. \& Gong, J. P. Effect of polymer entanglement on the toughening of double network hydrogels. J. Phys. Chem. B. 109, 16304-16309 (2005).

76 Liang, S., Yu, Q. M., Yin, H., Wu, Z. L., Kurokawa, T. \& Gong, J. P. Ultrathin tough double network hydrogels showing adjustable muscle-likeisometric force generation triggered by solvent. Chem. Commun. 48, 7518-7520 (2009).

77 Bin Imran, A., Seki, T., Kataoka, T., Kidowaki, M., Ito, K. \& Takeoka, Y. Fabrication of mechanically improved hydrogels using a movable cross-linker based on vinyl modified polyrotaxane. Chem. Commun. 41, 5227-5229 (2008).

78 Bin Imran, A., Seki, T., Ito, K. \& Takeoka, Y. Poly( $N$-isopropylacrylamide) gel prepared using a hydrophilic polyrotaxane-based movable cross-linker. Macromolecules 43, 1975-1980 (2010).

79 Bin Imran, A., Seki, T., Ito, K. \& Takeoka, Y. Hydrophobic and hydrophilic polyrotaxane based movable cross-linkers for thermo-sensitive poly( $N$-isopropylacrylamide) gels. Transactions of the MRS-J 35, 291-297 (2010). 


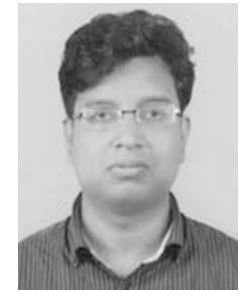

Abu Bin Imran was born in Comilla, Bangladesh in 1980. He received his Bachelor of Science and Master of Science degrees from the Department of Chemistry, Shahjalal University of Science and Technology, Sylhet, Bangladesh, in 2004 and 2006, respectively. He received his PhD degree from the Department of Molecular Design and Engineering of Nagoya University, Nagoya, Japan under the supervision of Professor Yukikazu Takeoka, in 2009. He has now been working as a post-doctoral fellow in the same group. He received the award of Material Back casting Technology (MBT) center research encouragement in 2010. His current research interests focus on smart hydrogels, photonic band gap materials and supramolecules.
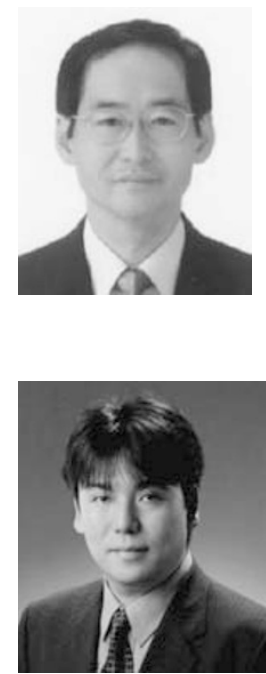

Yukikazu Takeoka was born in Osaka in 1968. He received his Bachelor and Master degrees from Sophia University. His first paper titled 'Electrochemical control of drug release from redox-active micelles' in the Journal of Controlled Release, written while pursuing his Master's degree, was awarded the 1995 Jorge Heller Journal of Controlled Release Outstanding Paper Award. He earned his Ph.D. in 1996 from Sophia University under the supervision of Professor Naoya Ogata, working on the electrochemical studies of redox-active supramolecules. He then joined Professor Toyoichi Tanaka's research group of the Massachusetts Institute of Technology with the Uehara Memorial Foundation for Postdoctoral Fellowships and worked on the phase transition of polymer gels and the synthesis of molecular recognition gels. His discovery of the phase transition of gels in which electrostatic interaction is the major attraction completes the list of phase transitions induced by all the fundamental chemical/biological interactions. In mid-1998, he moved to Yokohama National University, where he is a research associate. Since 2004, he has been a member of the group of Professor Seki at Nagoya University as an associate professor. His research interests focus on smart soft materials such as structural colored gels, high mechanical strength gels and densely grafted polymer brushes. 